\title{
The Impact of the Corona Pandemic On the Expansion of External Borrowing in Egypt
}

\author{
Shady Ibrahim Hassan Shehada \\ Economics Department, Faculty of Politics and Economics, Suez University, Arab Republic of Egypt \\ shady79shehada@yahoo.com
}

\begin{abstract}
:
External borrowing is one of the alternatives to help countries, especially developing ones, to overcome capital scarcity, and in light of the spread of the Corona pandemic that threatens both developed and developing economies. Most developing countries have turned to external borrowing to deal with the repercussions of the Coruna epidemic. Egypt, like other countries affected by the Corona pandemic, therefore this study aimed to study the impact of the Corona pandemic on the expansion of external borrowing in Egypt, where the researcher focused on the size of external loans before and after the pandemic. To achieve this object, the researcher relied in his methodology on the descriptive analytical approach through dealing with information and studies on the subject of the research, with the use of the quantitative approach in presenting data and analyzing it. The study concluded that the Corona pandemic had negatively affected government revenues from foreign exchange in Egypt, which led to a decline in the Egyptian foreign reserve, which leads to expansion in external borrowing and more foreign loans.
\end{abstract}

Keywords: External Borrowing; Corona Pandemic; Foreign Loans; Foreign Exchange Reserves; Egypt. 


\section{تأثير جائحة كورونا على التوسع في الاقتراض الخارجي في مصر \\ شادي إبر اهيم حسن شحاده \\ قسم الاقتصاد، كلية السياسة والاقتصاد، جامعة السويس، جمهورية مصر العربية \\ shady79shehada@yahoo.com}

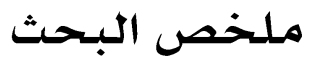

يُعد الاقتراض الخارجي أحد البدائل لمساعدة الدول وبخاصة النامية منها في التغلب على ندرة رأس المال، وفي ظل إنتشار جائحة كورونا التي تهدد الاقتصاديات المتقدمة والنامية على حد سواء. توجهت معظم الدول النامية نحو الاقتراض الخارجي لمواجه تداعيات وباء كورونا. ومصر كغيرها من الدول التي تأثرت بجائحة كورونا، لذلك هدفت هذه الدراسة إلى دراسة تأثير جائحة كورونا على التوسع في الاقتراض الخارجي في مصر، حيث ركز الباحث على حجم القروض الخارجية قبل الجائحة وبعدها. ولتحقيق ذلك المدف إعتمد الباحث في منهجيته على المنهج الوصفي التحليلي من خلال تناول المعلومات والدراسات الخاصة بموضوع البحث، مع الإستعانة بالمنهج الكمي في عرض البيانات وتحليلها. وقد توصلت الدراسة إلى أن جائحة كورونا أثرت سلباً على الاير ادات الحكومية من النقد الاجنبي في مصر مما أدى إلى تراجع الاحتياطي الأجنبي المصري، مما سيؤدي إلى التوسع في الاقتراض الخارجي ومزيد من القروض الخارجية.

الكلهمات المفتاحيـة: الاقتراض الخارجي، جائحة كورونا، القروض الخارجية، الإحتياطي النقدي الأجنبي، مصر. 


\section{المقدمة}

يُعد الاقتراض الخارجي أحد مصادر التمويل للدول نتيجة عجز الموارد المحلية أو قصور حجم المدخرات الوطنية عن تغطية الاستثمارات المطلوبة، وحاجة الدولة للعملات الصعبة، وفي ظل الإنتشار الكبير لجائحة كورونا جعلت الاقتصاديات الدولية في حالة عدم اليقين، حيث باتت تشكل مصدراً من مصادر التقلبات، وعدم الإستقرار الاقتصادي، ومن المتوقع أن يكون للجائحة تأثيرات كبيرة على الاقتصاديات المتقدمة و النامية. خاصة ما تعلق برصيد راس المال من العملات الأجنبية، لذلك توجهت معظم الدول النامية نحو الاقتر اض الخارجي لمواجه تداعيات وباء كورونا. والاقتصاد المصري ليس بعيداً عن التداعيات التي ثُلاحق الاقتصاديات العالمية بسبب تطور هذا الوباء، فمع إستجابة مصر للإجراءات الإحترازية والوقائية وتوقف حركة النقل والسفر أدى ذلك إلى إنكماش إيرادات الدولة الحكومية من النقد الأجنبي بسبب التراجع في إيرادات السياحة، إيرادات قناة السويس، تحويلات العاملين بالخارج، خروج الأموال الساخنة من مصر، بالأضافة إلى تراجع الاستثمار الأجنبي المباشر. مما أدى ذلك إلى تراجع الإحتياطي النقدي الأجنبي المصري من 46 مليار دولار في فبراير 2020 إلى 36 مليار دولار في نهاية مايو 2020 2، وفقاً لإحصائيات البنك المركزي المصري، مما سيؤدي إلى رفع سقف الاقتراض الخارجي

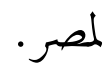
وعليه فإن مشكلة الدراسة تتمثل في التساؤل حول: ما مدى إنعكاس جائحة كورونا على التوسع في الاقتراض الخارجي في مصر؟ وترتكز الدراسة على فرضية أساسية مؤداها: قد تساهم جائحة كورونا في التوسع في الإقتراض الخارجي في مصر. - (- مص وتهدف الدراسة إلى دراسة اثر إنعكاس جائحة كورونا على التوسع في الاقتراض الخارجي في مصر، حيث يركز الباحث على حجم القروض الخارجية قبل الجائحة وبعدها. 
وتنبع أهمية الدراسة في أن التوسع في الاقتراض الخارجي سيؤثر بشكل كبير على قيمة العملة المحلية أمام العملات الأجنبية، بالاضافة إلى إرتفاع معدلات التضخم، وعجز الموازنة العامة للدولة. وتعتمد منهجية الدراسة على المنهج الوصفي التحليلي من خلال تناول المعلومات والدراسات الخاصة بموضوع البحث، مع الإستعانة بالمنهج الكمي في عرض البيانات وتحليلها.

\section{اولاً : الاقتراض الخارجي مفهومه - أشكاله - تداعياته}

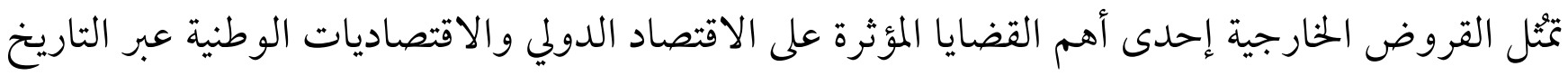

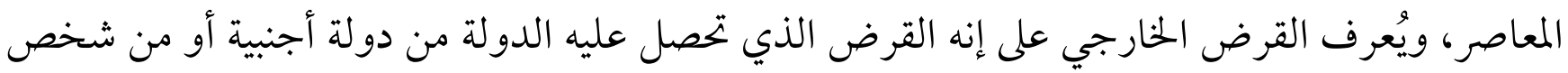
طبيعي أو إعتباري مقيم في الخارج أو من هيئة حكومية أو صندوق حكومي أو دولي دلي أو منظمة دولية في

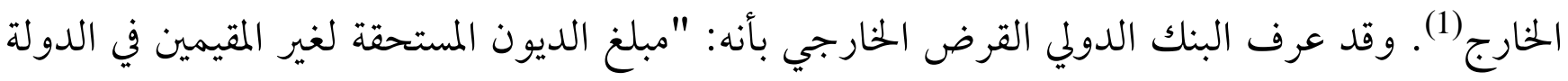

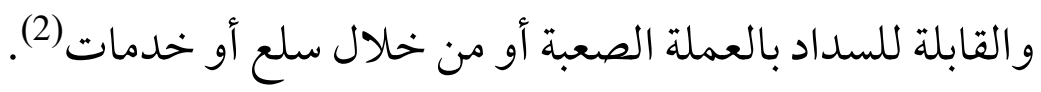
كما يمكن تعريفه وفق العديد من المنظات الاقتصادية الدولية مثل منظمة التعاون الاقتصادية للتنمية، وصندوق النقد على إنه "الدين الإجمالي في تاريخ معين، والذي يعادل مبالغ الالتزامات التعاقدية الجحارية

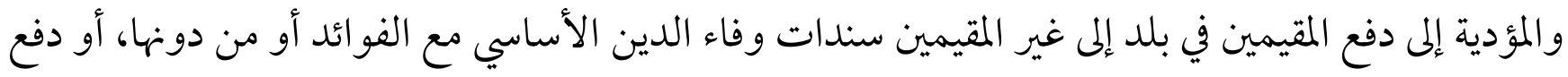
الفائدة مع سداد المبلغ آو من دونه (3). ويمكن تعريف القروض الخارجية من وجه نظر الباحث على إنها المبالغ التي تظهر في جانب الايرادات في الموازنة العامة للدولة عند الحصول عليها، وفي نفس الوقت هي المبالغ التي تظهر في جانب النفقات في الموازنة العامة عند سداد الاقساط والفوائد الناتجة عنها. وتتمثل أهم أشكال القروض الخارجية في مايلي (4): 
1) القروض التجارية التي تعقدها الدول النامية، والتي تقع ضمن الدول ذات الدخل المنخفض والمتوسط مع أسواق المال الدولية والمصارف الدولية عابره الجنسيات وجهات مقرضه أخرى تسمى بالدائنين الخو اص.

القروض الميسرة التي تقدم بطريقة ثنائية أو متعددة الأطراف كقروض رسمية من دول مانحة يجمها نادي باريس ولجنة المساعدات في منظمة التعاون الاقتصادي والتنمية، إلى جانب المنظات الدولية كالبنك الدولي وصندوق النقد الدولي. إصادر السندات التي تبيعها الدول المدينة كصكوك مالية ترتب حقوقاً مالية على شكل فوائد مدفوعة للدائنين من مشترى السندات عبر أجال زمنية محددة. 4) تدفقات روؤس الاموال في صورة حقوق ملكية أو أسهم من خلال الاستثارات الأجنبية كالاستثمارات المباشرة بنسية مشاركة 10 \% أو أكثر، الاستثمارات غير المباشرة بمثلة في استثمارات الحوافظ المالية عبر اسواق المال بأي نسبة. أما عن تداعيات القروض الخارجية، فالقروض الخارجية لها بجموعة من التداعيات نذكر منها: 1) في الاجل القصير تعمل القروض على زيادة عرض العملة الأجنبية، مما يؤدي إلى حدوث رواج اقتصادي وزيادة الاحتياطي النقدي الأجنبي للدولة. في الأجل الطويل تؤدي القروض إلى حدوث عجز بالموازنة العامة للدولة، التضخم، وتدهور معدل الصرف مما سيؤثر على قيمة العملة الوطنية. بل يحدث انكماش اقتصادي نتيجة نقص عرض العملة الأجنبية حيث يتم التوجه لسداد الاقساط والفوائد الناتجة عن الاقتراض الخارجي، مما يؤدي لإنخفاض النمو الاقتصادي. 3) تشكل القروض الخارجية عبئًا نقدياً على الاقتصاد الوطني ككل، فالاجيال القادمة هي من تسدد فاتورة المدفوعات، إضافة إلى إنه يشكل عبئًا على ميز ان المدفوعات (5). 


\section{ثانيًا : وضع الاقتصاد المصري قبل جائحة كورونا وبعدها}

\section{أ- وضع الاقتصاد المصري قبل جائحة كورونا}

وفقاً لصندوق النقد الدولي، كانت مصر تشهد اتجاهاً اقتصادياً إيجابياً قبل تفشي المرضك، فقد بلغ بلغ معدل نمو الناتج المحلي الإجمالي نسبة 5.5\% في 2018/20192، وكانت التقديرات أكثر إيجابية لعام 2019 / 2020 ليحقق نسبة 5.8 \%، و كان من المتوقع أن يصل إلى 6.0 \% لعام 20212021 / 20 ـ إضافة إلى إنخفاض معدل البطالة في البلاد لنسبة 8.8 \% خلال 8018 / 2019 (وهو أدنى مستوى له منذ أكثر من عقد)، وإنخفاض في الضغط التضخمي، بل إن الميزانية كانت تحت السيطرة. وعلى الرغم من انخفاض نسبة الدين إلى الناتج المحلي

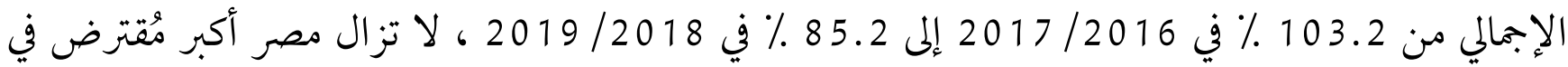

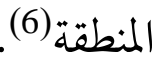
بل إن التوقعات الاقتصادية العالمية لصندوق النقد الدولي، كانت تشير إلى أنه من المتوقع أن تكون مصر الدولة الوحيدة في منطقة الشرق الأوسط وشمال إفريقيا التي تشهد نمواً اقتصادياً إيبابياً في عام 2020 2، بنسبة قدرها 95 . 1.9 ف، فين أن جميع الاقتصاديات الأخرى في المنطقة من المتوقع أن يتقلص نموها الاقتصادي. والشكل التالي يوضح معدل النمو لمصر خلال الفترة من 2015- 20192، والتوقعات خلال 2020، 2021 202. شكل (1 ): معدل نمو الناتج المحلي الإجمالي المصري خلال الفترة 2015 - 2021

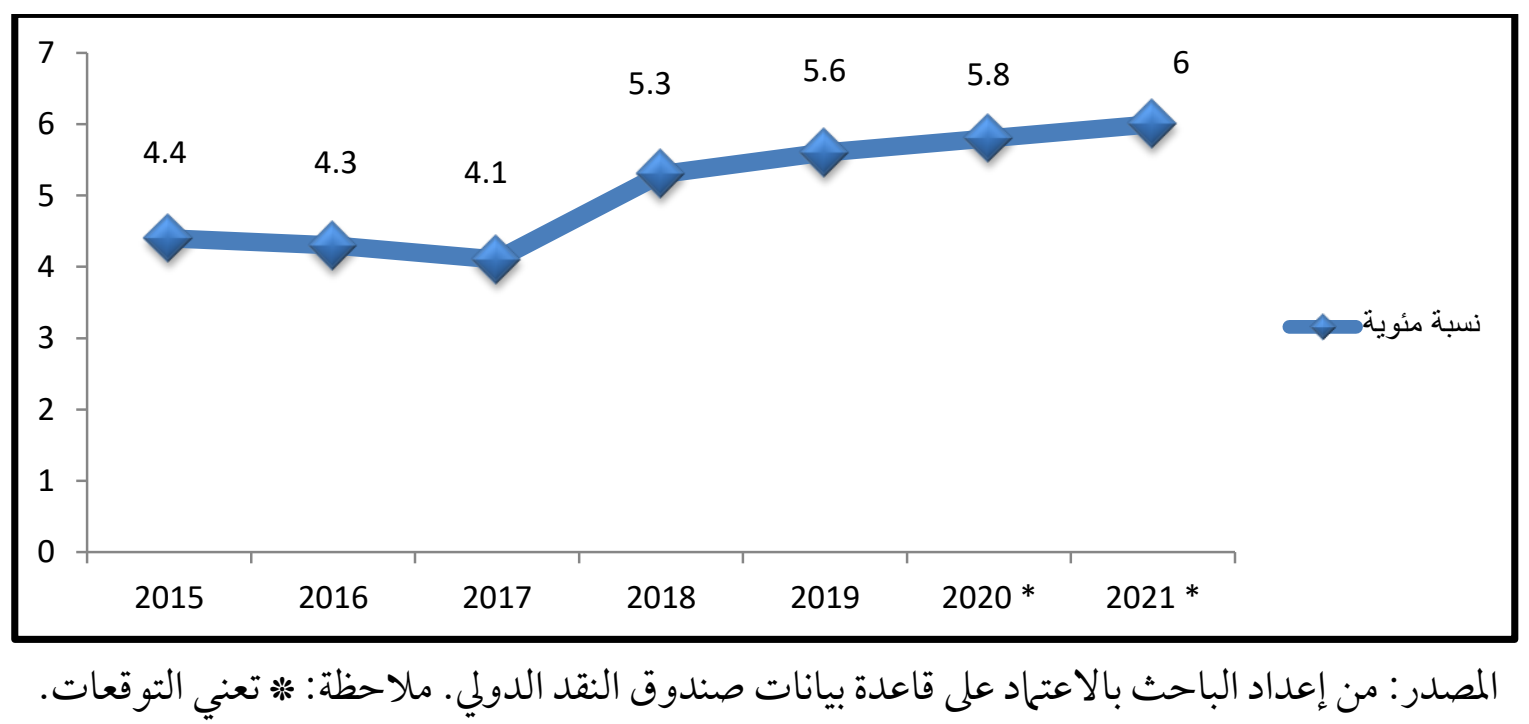


ولقد ساعد تحسن مؤشرات الاقتصاد المصري في تحقيق مراكز متقدمة في المؤشرات الدولية، فقد أشادت "فاينشال تايمز" في 190 بتحسن أداء قطاع السياحة في مصر مدفوعاً بالأسعار المنخفضة وتحسن الأحوال الأمنية. كما أشارت قائمة "إيكونوميست"، الصادرة عن مؤسسة فيتش سوليوشنز في الربع الثالث لعام 2019 إلى تحسن معدلات النمو الاقتصادي، حيث احتل النمو الاقتصادي المصري المركز الثالث على مستوى العالم(7). بل كانت تتوقع استمرار النمو الاقتصاد المصري في تحقيق أفضل أداء له في منطقة الشرق الأوسط وشمال أفريقيا للعام الملالي 2019 / 2020. أما تقرير الأونكتاد (مؤتمر الأمم المتحدة للتجارة والتنمية)، فقد أشار إلى تحسن أداء الاستثمار في مصر، مشيراً إلى استمرار مصر في صدارة الدول الأفريقية الجاذبة للاستثمار الأجنبي المباشر خلال النصف الأول من عام 2019(8). وفي تقرير ممارسة الأعمال 2020 الصادر عن البنك الدولي أشار إلى تحقيق مصر المركز الـ114(9) وهو مركز متقدم، مقارنة بالمركز الـ 120 في تقرير ممارسة الأععال 2019 (10) بمعدل تغيير قدره (+6). وحققت مصر المرتبة الـ 63 بتقرير التنافسية العالمي عام 2019 في مؤشر شفافية الموازنة، مقارنة بالمرتبة الـ 110 بتقرير عام 2018 وبذلك تكون قد تقدمت مصر (+7 4) مركزاً، وكذلك تقدمت (+18) مركزاً في مؤشر معيار التدقيق والمحاسبة، لتحتل المرتبة 50 بتقرير التنافسية العالمي عام 2019، مقارنة بالمرتبة 68 بتقرير عام 2018 (11)

وشهد التصنيف الائتماني لمصر تحسن في أبرز وكالات التصنيف العالمية، فوفقاً لو كالة "فيتش" ارتفع التصنيف الائتحاني لمصر من B إلى B+ مع نظرة مُستقبلية مستقرة، وكذلك ارتفع التصنيف الائتحاني لمصر من B3 إلى B2، وذلك وفقاً لو كالة "موديز"، في حين أبقت وكالة "ستاندرد آند بورز" على التصنيف الائتحاني لمصر عند B مع نظرة مستقبلية مستقر، ويمكن بيان ذلك على النحو التالي (12): 
جدول (1 ): تقييم الو كالات العالمية للتصنيف الائتماني لمصر

\begin{tabular}{|c|c|c|c|c|c|}
\hline \multirow[b]{2}{*}{ الأفاق المستقبلية } & \multicolumn{2}{|c|}{ التاريخ } & \multicolumn{2}{|c|}{ التقييم } & \multirow[b]{2}{*}{ 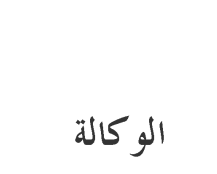 } \\
\hline & السابق & الحالي & السابق & الحالي & \\
\hline مستقر & $2018 / 8 / 28$ & $2019 / 4 / 17$ & B3 & $\mathrm{B} 2$ & موديز \\
\hline مستقر & $2018 / 1 / 16$ & $2019 / 5 / 21$ & B & $\mathrm{B}_{+}$ & فيتش \\
\hline مستقر & $2017 / 11 / 10$ & $2018 / 5 / 11$ & B- & B & ستاندرد آند بورز \\
\hline
\end{tabular}

Tradinge Economics. المصدر:من إعداد الباحث بالاعتهاد على وكالات التصنيف العالمية و

\section{ب- وضع الاقتصاد المصري بعد جائحة كورونا}

من المتوقع أن ينكمش النشاط الاقتصادي في الاقتصادات المتقدمة بنسبة 7 \% في عام 2020 من جراء الاضطر ابات الشديدة التي أصابت جانبي الطلب والعرض المحليين، والتجارة، والتمويل. وتشير التنبؤات إلى أن اقتصاديات الأسواق الصاعدة والبلدان النامية ستشهد انكماشاً نسبته 2.5 ٪ في 2020، وهو أول انكماش لها كمجموعة منذ 60 عاما على الأقل. ومن المتوقع أن ينخفض متوسط نصيب الفرد من الدخل بنسبة 3.6 ٪، متسبباً في سقوط ملايين من الناس في براثن الفقر المدقع هذا العام(13). وستكون الدول الأكثر تضرراً من صدمة [كوفيد 19] هي الدول التي كانت فيها الجائحة أشد تأثيراً، والتي تعتمد اعتهاداً كبيراً على التجارة العالمية أو السياحة أو صادرات السلع الأولية، والتمويل الخارجي. وبالرغم من إختلاف حجم إضطراب النشاط الاقتصادي من منطقة إلى أخرى، تعاني كل اقتصاديات الأسواق الصاعدة والبلدان النامية من مواطن ضعف تفاقمت بسبب صدمات خارجية. علاوةً على ذلك، من المرجح أن تُخلِّف الاضطر ابات في خدمات التعليم، وتعذر الحصول على الرعاية الصحية الأولية، آثار ادائمة على تنمية رأس المال البشري. أما على مستوى الاقتصاد المصري، فإن الأمر أكثر تعقيداً لمصر كجزء من هذا المشهد العالمي والإقليمي فنجد 
البلاد تشهد حالياً برنابجاً جذرياً لإصلاح المشكلات النقدية والمالية القديمة بالإضافة إلى استمرار

$$
\text { الاختلالات الهيكلية. }
$$

تنطوي هذه الأزمة على العديد من القطاعات الضعيفة، مثل السياحة والتدفقات المالية الأجنبية، بالإضافة إلى قطاع التصنيع الذي يعتمد بشكل كبير على المواد الخام المستوردة ويشارك بعمق في

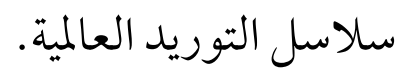

تعرضت مصر بشكل مفرط لحلقات مستمرة من الاضطرابات السياسية الداخلية، بدءاً من ثورة 2011، من خلال مجموعة من الدورات السياسية المتتالية وانتهاءً بالتوترات المصرية الإثيوبية

المتصاعدة مؤخراً.

$$
\text { لا تزال البلاد تكافح مع الوضع الاجتماعي والاقتصادي. }
$$

وما زالت تعاني من ضعف المصداقية والأداء المؤسسي المتواضع الذي يؤثر على المتغيرات الاقتصادية

$$
\text { والتوقعات العامة ، وخاصة في أوقات عدم اليقين المفاجئ. }
$$

وتشير تقديرات المركز المصري للدراسات الاقتصادية إلى أن آثار Covid-19 على الاقتصاد المصري من المرجح أن تكون ملموسة حتى يونيو 2021(15)، فعلى الرغم من أن الاقتصاد المصري حقق تقدماً بنسبة 5 \% على أساس ربع سنوي في الربع الأول من عام 2020، مقارنة بـ 5.6 ٪ في الفترة السابقة(16). فإن تقرير صادر عن وزارة التخطيط والتنمية الاقتصادية يبين أن إجراءات الاحتواء العالمية للجائحة والتي بدأت السلطات المصرية بفرضها وإتباعها في مارس 2020، تسببت في تباطؤ الأنشطة الاقتصادية، بل من المتوقع أن يبلغ معدل النمو السنوي للناتج المحلي الإجمالي في مصر 2.50 ٪ بحلول نهاية هذا الربع، ومن المتوقع أن يُقدر معدل النمو السنوي للناتج المحلي الإجمالي في مصر ليبلغ 2.70 ٪٪ في نهاية 2020 . على المدى الطويل، وأن يتجه معدل النمو السنوي للناتج المحلي الإجمالي في مصر إلى حوالي 40 \% ف في عام 2021 و 4.60 \% في عام 2022 2017). و الشكل التالي يبن تطور الناتج المحلي الإجمالي على أساس ربع سنوي. 
شكل (2) معدل النمو للناتج المحلي الإجمالي في مصر ريع سنوي

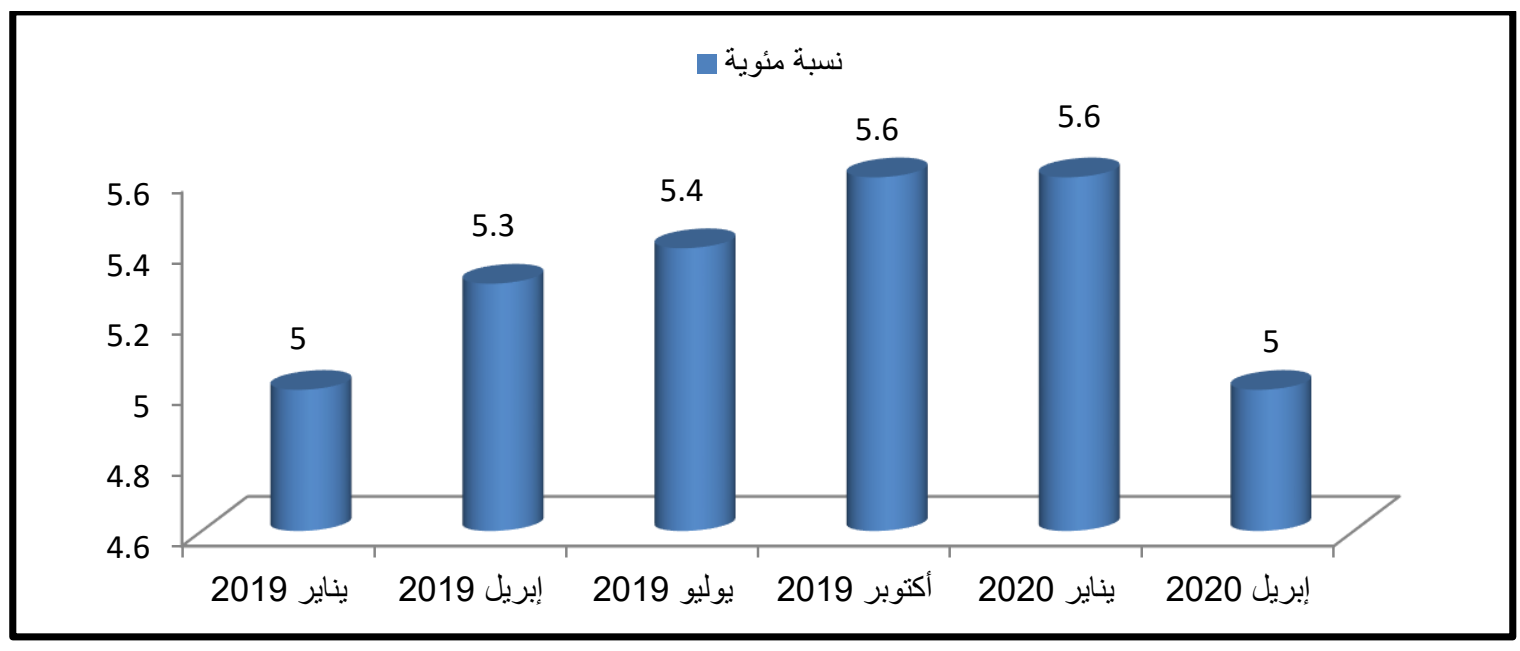

https://tradingeconomics.com/egypt/gdp-growth-annual المصدر: من إعداد الباحث بالاعتماد على

\section{ثالثًا : تطور القروض الخارجية المصرية خلال السنوات الأخيرة}

بنظره عامة على تقرير إحصائيات القروض الدولية لعام 2020 الصادر عن البنك الدولي، تشير البيانات إلى أن إجمالي القروض الخارجية للبلدان منخفضة الدخل ومتوسطة الدخل قفزت بنسبة 5.3 ٪ إلى 8.8 تريليون دولار العام الماضي، وأن صافي تدفقات الديون (إجمالي المدفوعات مطروحا منها أقساط سداد أصل القروض) من الدائنين الخارجيين هبطت 28 ٪ إلى 529 مليار دولار. يشير التقرير إلى أنه في المتوسط، كان عبء الديون الخارجية للبلدان منخفضة الدخل ومتوسطة الدخل معتدلا، لكن عدة بلدان شهدت تدهور مسار ديونها منذ عام 200 ـ وتقلصت نسبة البلدان منخفضة الدخل ومتوسطة الدخل التي تقل فيها نسبة الديون إلى إجمالي الدخل القومي عن 30 ٪ إلى 25 ٪٪ مقابل 42 \%٪ قبل عشر سنوات. وبالمثل قفزت نسبة البلدان التي ترتفع فيها نسبة الديون إلى الصادرات(18).

وتعود قضية الاقتراض الخارجي في مصر خلال السنوات الأخيرة إلى عدد من الاسباب مثثلة في (19): 1) طبيعة استخدام القروض الخارجية والتي تُعد من أسباب تفاقم مشكلة القروض، حيث توجه نسبة من القروض إلى تمويل عجز الميزانية، واستيراد السلع الاستهلاكية، وخصوصاً المو اد الغذائية، ويوجه جزء 
آخر إلى تمويل مشروعات إنتاجية عائدها لا يغطي تكاليف الاقتراض، ومن ثم فقد أصبحت القروض عبئًاً إضافياً على الاقتصاد المصري. الارتفاع الكبير في أسعار الفائدة في أسو اق المال الدولية، الأمر الذي أدى إلى زيادة المديونية، إذ تجاوزت في العديد من البلدان الفائدة المدفوعة قيمة التمويل الإضافي، وأصبحت خدمة الدين تمثل مقداراً كبيراً من الدين، وتستحوذ على مبالغ كبيرة من النقد الأجنبي.

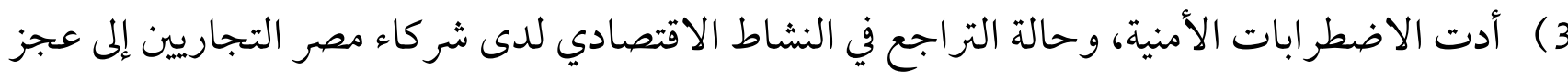
في ميزان المدفوعات، الأمر الذي أدى إلى انخفاض حصيلتها من النقد الأجنبي، وقد اعتمد عليها الاقتصاد المصري في تكوين احتياطياته من النقد الأجنبي، كقطاع السياحة والصناعة وغيرها. 4) تراجع التدفقات الرأسمالية نتيجة لتمويل الأنشطة الجارية بالعملة الأجنبية، عن طريق السحب من رصيد الاحتياطي مـن العملات الأجنبية لدى البنك المركزي. وعن تطور القروض الخارجية المصرية في السنوات الأخيرة نجد أن:

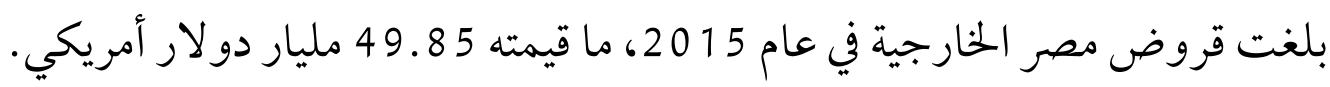

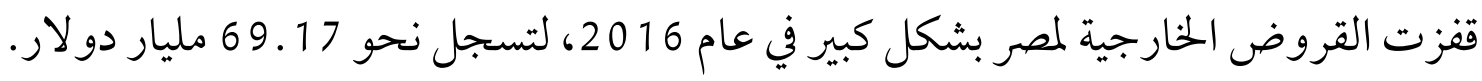

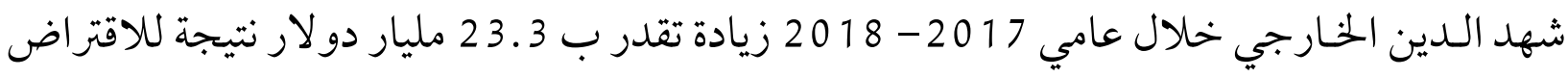
من خلال اصدار سندات دولارية بقيمة 7 مليار دولار (20). من المتوقع أن يصل حجم القروض الخارجية لمصر بنهاية 2020، 2021 إلى 116 مليار، 120 مليار دولار أمريكي على التوالي. والشكل التالي يوضح التطور السنوي للقروض الخارجية المصرية، والتوقعات خلال 2020، 2021. 
شكل (3): تطور القروض الخارجية المصرية خلال الفترة 2015 - 2021 مليار دولار أمريكي

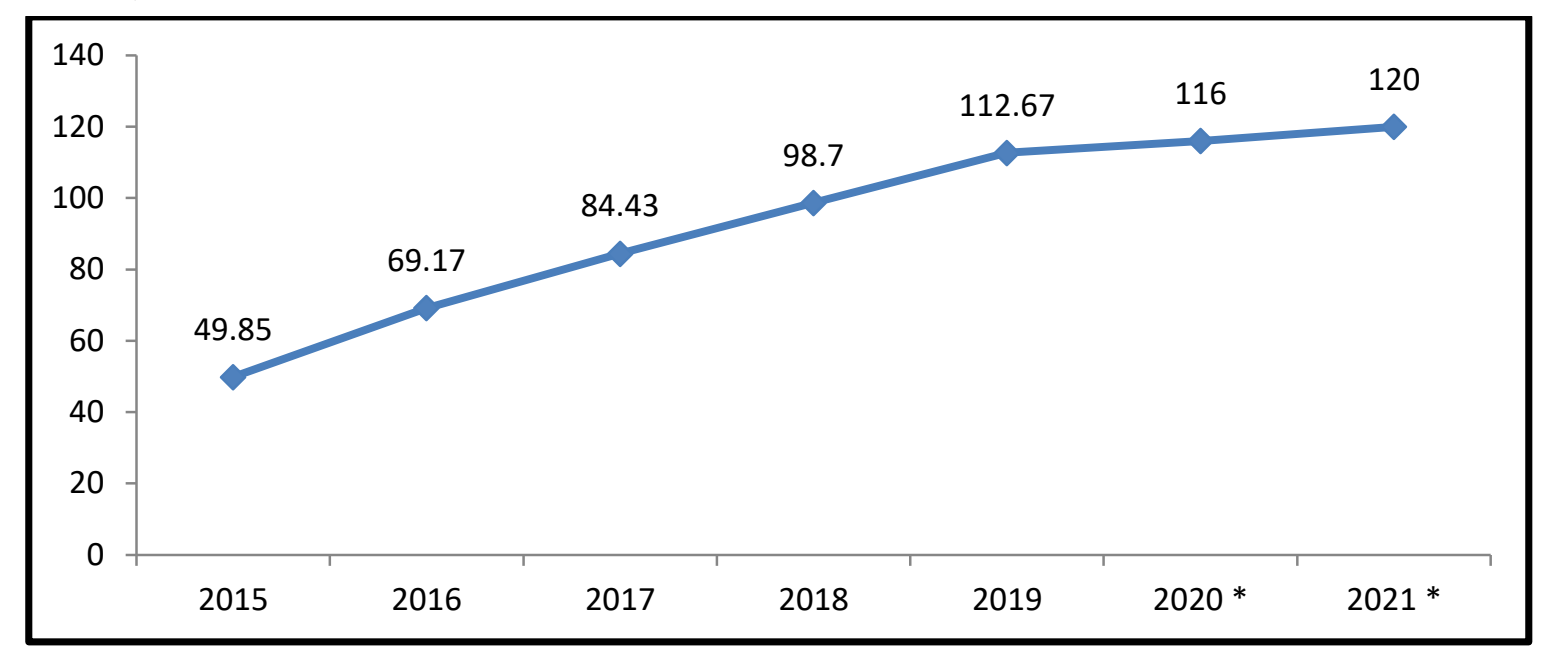

المصدر: من إعداد الباحث بالاعتلاد على بيانات البنك الدولي و .Trading Economics ملاحظة: قتعني التوقعات

فقد بدات مصر برنامج للاصلاح الاقتصادى من صندوق النقد الدولي في نوفمبر 2016، حصلت بموجبه على قرض بقيمة 12 مليار دولار، يتم إستلامه على دفعات خلال ثلاث سنوات وقد حصلت مصر على الدفعة الأخيرة منه في يونيو 190 2. وتعهدت مصر بعدم اللجوء مجدداً إلى صندوق النقد الدولي، وعليها أن تسدد القسط الأول من القرض علاوة على فائدة لا تزيد على 2 \% خلال النصف الأول من 2021، بعد استنفاذ فترة السماح عقب حصولها على الشريحة الأولى من التمويل. إلا أن شراسة جائحة كورونا، اضطرت الدولة المصرية إلى تنفيذ خطة احترازية شاملة، بلغت تكلفتها 100 مليار جنيه مصري(21). بها يعادل (6.3 مليار دولار أمريكي)(22) بهدف تخفيف أثر الأزمة إلى أقل حد ممكن، ومعالجة التداعيات الاقتصادية السلبية، الناجمة عن توقف الإنتاج، وتعليق حركة الطيران، وتقييد حركة الأفراد. ومع الإجراءات الاحترازية المشددة، التي تهدف إلى الحفاظ على النظام الصحي من الانهيار، فقدت الخزانة المصرية أهم مواردها من السياحة نتيجة توقف 400 ألف سائح شهرياً كانت تستقبلهم المنتجعات السياحية، بالإضافة إلى فقد الآلاف من الذين يعملون في الخدمات السياحية وظائفهم، تحويلات المصريين العاملين 
بالخارج بسبب تراجع أصحاب العمل عن المشاريع و / أو تسريح الموظفين ما سينعكس على القوة الشرائية

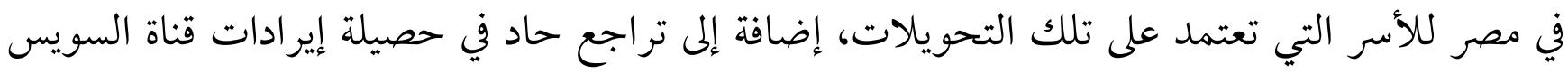

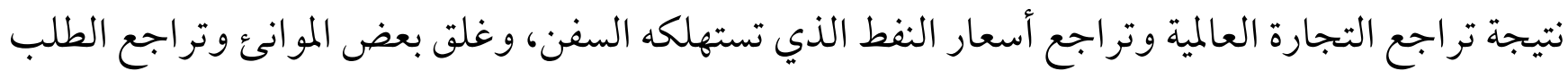

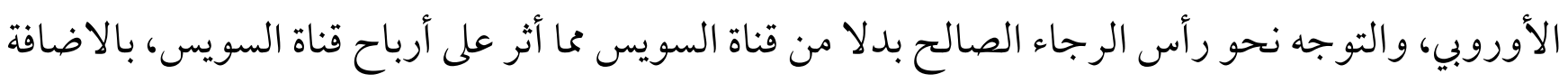
إلى إنخفاض صادرات مصر نتيجة توقف حركة النقل عبر الحدود الدولية. كما ساهمت تدفقات رأس المال الخارجة من استثمارات الأجانب في المحافظ المالية بقيمة تتراوح بين 2 إلى 2.5 مليار دولار (23)، في ظل بحث المستثمرين عن ملاذات استثمارية آمنة في ظل تقلبات الأسواق، فمن المتوقع أن تستخدم السلطة النقدية احتياطاتها للحد من الانخفاضات الكبرى في قيمة الجنيه، ومع استمرار الأزمة ستزيد المسحوبات من الأذون مما يعني تبخر الاحتياطي وعدم قدرته على إيقاف نزيف قيمة الجنيه المصري.

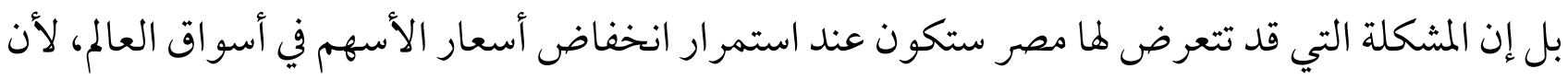

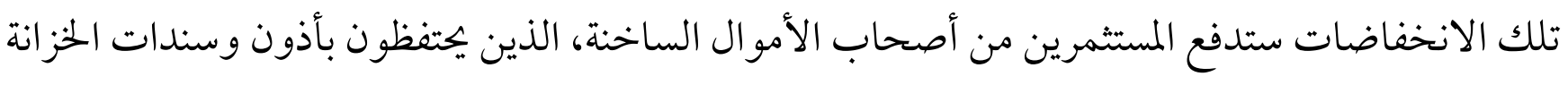
بالجنيه المصري، إلى تسييل محافظهم الرابحة، مما يعني توجهوم لشراء الدولار الذي باعوه عند دخولهم،

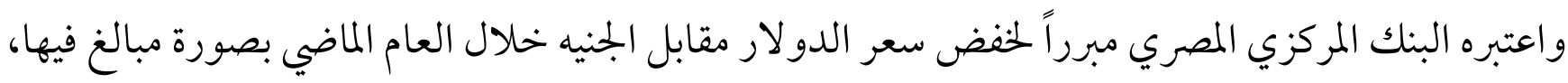
رغم الطبيعة غير المستقرة لتلك الاستثمارات، ما يعني المزيد من الضغط على سعر الصرف.

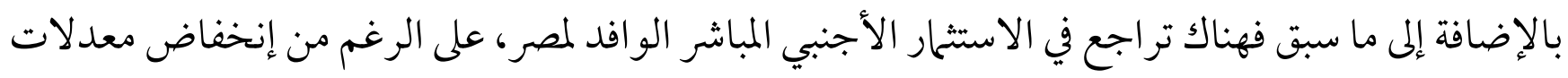
الفائدة في مصر خلال عام 2020 بمعدلات ملموسة، كجزء من حزمة تنشيطية لمساعدة الإقتصاد الوطني للخروج من ركوده نتيجة الإضرابات في النشاط الإقتصادي العالمي في أعقاب تفشي فيروس كورونا، ما سيساهم في خسارة خزانة الدولة المصرية للعملة الأجنبية. لقد أدت تلك العوامل إلى إنخفاض حصيلة النقد الأجنبي ومن ثم أثرت على الإحتياطي النقدي الأجنبي المصري مما دفع مصر نحو الاقتراض الخارجي من جديد، لتعلن مصر في إبريل 2020، طلب تمويل إضافي من 
صندوق النقد الدولي، بواسطة آلية التمويل السريع، التي أتاحها الصندوق لكل الدول الأعضاء، التي تواجه احتياجات طارئة.

وقد تسلمت مصر في مايو 2020 من صندوق النقد الدولي قرضاً بقيمة 2.7 مليار دولار، وهو ما يعادل نحو 100 ٪ من حصتها وفقاً لأداة التمويل السريع، والتي تستهدف التعامل مع الصدمة الناتجة عن فيروس كورونا المستجد (24).

ونتيجة إنخفاض الايرادات الحكومية المصرية من النقد الأجنبي حيث تراجع الاحتياطي النقدي الاجنبي المصري بها يعادل 10 مليار دولار خلال ثلاث شهور ( مارس - إبريل - مايو) من 2020، فمن المتوقع أن تطلب مصر قروضاً جديدة من الاسواق العالمية وصندوق النقد الدولي بقيمة 13 مليار دولار أمريكي، حيث من المتوقع أن تقترض من صندوق النقد الدولي بما قيمته 5.3 مليار دولار أمريكي (25). لتصل حصيلة ما تقترضه مصر من صندوق النقد الدولي ما يعادل 20 مليار دو لار أمريكي في أقل من خمس سنوات، والشكل التالي يبين تراجع الاحتياطي النقدي المصري.

شكل (4) الإحتياطي النقدي الاجنبي المصري على أساس شهري

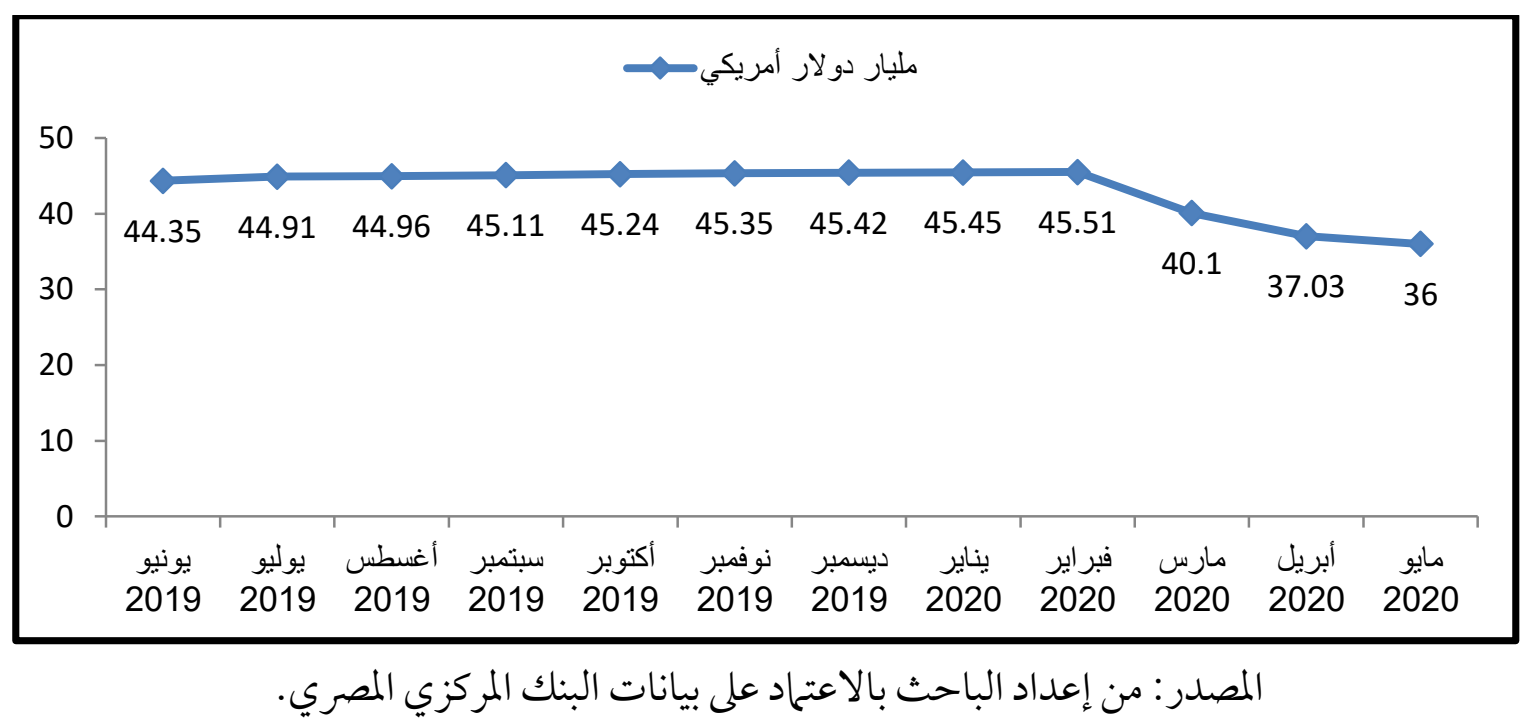


وقد أرجع البنك المركزي المصري التراجع في الاحتياطي النقدي الأجنبي، إلى استخدم نحو 8.5 مليار دولار منه في تغطية احتياجات السوق المصرية من العملات الأجنبية، وتغطية تراجع استثمارات الأجانب، و المحافظ الدولية، وضمان استيراد السلع الاستراتيجية، بالإضافة إلى سداد الالتزامات الدولية الخاصة بالديون الخارجية على الدولة. إن توجه مصر نحو التوسع في الاقتراض الخارجي وبخاصة صندوق النقد الدولي سيكون له أثار سلبية على المواطنين وعلى موارد الدولة نفسها، فالقروض ستؤدي إلى مزيد من فرض الضرائب و الرسوم الجلديدة، وهذا ما حدث بالفعل حيث قامت الحكومة بتحرير دعم الكهرباء عما كان عليه في 2019، لتبدا مع يوليو 2020 بتطبيق أسعار جديدة، إضافة إلى رفع رسوم بعض المعاملات الحكومية، بالاضافة إلى تراجع في قيمة الجنية

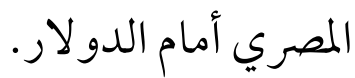
اما على مستوى موارد الدولة فإن القروض الجلديدة ستؤدي إلى إهدار الموارد المحلية لان ما سيتم تحقيقيه من مكاسب سيتحول إلى سداد الاعباء من الأقساط والفو ائد الناتجة عن هذه القروض.

تُعد مشكلة القروض الخارجية من المشاكل المعقدة التي تواجهها الدول النامية بشكل عام ومصر بشكل خاص، وفي هذه الدراسة حاول الباحث دراسة تأثير جائحة كورونا على التوسع في الاقتراض الخارجي في مصر، والوقوف على العوامل التي أدت إلى توجه مصر نحو التوسع في الاقتراض الخارجي من الاسواق العالمية ومن صندوق النقد الدولي. إن إنخفاض الايرادات الحكومية من العملة الصعبة نتيجة جائحة كورونا التي أدت إلى غلق تام وشبه كامل للنشاطات الاقتصادية عالمياً ومحلياً، جعلت حصيلة مصر من النقد الأجنبي تتراجع من 46 مليار دولار أمريكي في فبراير 2020 إلى 36 مليار دولار أمريكي في مايو 2020، وهذا التراجع كان نتيجه لعدد من العوامل وهي تراجع إيرادات السياحة، وتحويلات العاملين المصريين بالخارج، وأنخفاض إيرادات قناة 
السويس، وإنخفاض الصادرات المصرية، وخروج الاموال الساخنة من مصر، وتراجع الاستثمار الاجنبي

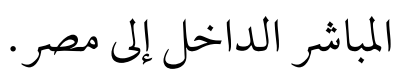

ما كان له الاثر في توجه مصر نحو التوسع في الاقتراض الخارجي في محاولة من السلطات المصرية الحفاظ على حصيلة النقد الاجنبي ومنع الحركة العشوائية للعملة الأجنبية، وفي مقابل ذلك سيخسر الجنية المصري المكاسب التي حققها في 190 2، إضافة إلى عدم إستقرار الموازنة العامة للدولة.

التوصيات:

توصلت الدراسة إلى أن جائحة كورونا ستساهم في توسع مصر في الاقتراض الخارجي نتيجة إنخفاض الاحتياطي النقدي المصري من العملات الأجنية، ومن ثم سيؤدي ذلك لزيادة حجم القروض الخارجية المصرية، مما يؤدي إلى زيادة الاعباء كفرض ضرائب جديدة على المو اطنين وإستنزاف للموارد المحلية للدولة، فالقروض الجمديدة تهدر ثروتنا القومية وتزيد معدلات الفقر، وتزيد من أثارها السلبية على الطبقات الفقيرة ومحدودي الدخل. لذلك يوصي الباحث في نهاية الدراسة بها يلي: 1) التوجه نحو دعم خطط إنتاجية من شأنها دعم قطاعات الإنتاج والمصانع في مصر لخفض معدلات الإستير اد من الخارج. 2) التوجه نحو ترشيد النفقات العامة الحكومية تعويضاً عن الاقتراض من الخارج حتى لاتزيد الأعباء والممثلة في الأقساط والفو ائد الناتجة عن الاقتراض الخارجي. 3) العمل على إستخدام القروض الجلديدة لتمويل مشروعات تنموية تعمل على خلق وتوسيع طاقات إنتاجية وليس للاستهلاك الجاري، لكي يتمكن الاقتصاد القومي من تدبير الموارد الضرورية لخدمة أعباء القروض الخارجية في الفترة المقبلة. 
(1) دوابه، أشرف محمد. أزمة الدين العام المصري رؤية تحليلية. بحلة المعهد المصري للدراسات الاقتصادية والسياسية، 2016م، العدد:

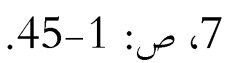

2015). The Impact of External Debt on Economic (Ahmed, Y. A., Saeed, S. T., \& Hama Saed, S. J. (2) Growth:Empirical Evidence from Iraq. International Journal of Science and Research (IJSR), 4(8), 1506-

(3) بوطيبه، فيصل. أثر التصحيح الهيكلي على المديونية الخارجية في الجزائر. رسالة ماجستير غير منشورة: كلية العلوم الاقتصادية والتسيير و العلوم التجارية، 2004م، الجزائر.

عيسى، محمد عبد الشفيع. أزمة الديون العالمية وبعض تأثيراها على التنمية الاقتصادية مع الاشارة إلى الاقتصاد المصري. معهد

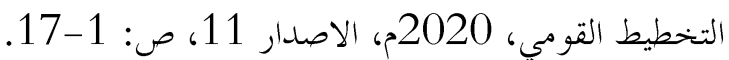

(5) المياحى، عمار فوزي. القروض الخارجية كأحد وسائل حل عجز الموازنة المستدام بالدول العربية النفطية وغير النفطية مصر والعراق

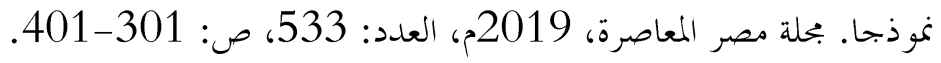
1-5.:OECD. (2020, April). The Covid-19 Crisis In Egypt. P (6) (7) سعيد، كريم. 2019م. كيف رأى العالم الاقتصاد المصري خلال 2019؟. تاريخ الاسترجاع: 26-سبتمبر -2019م.نشر بموقع: https://al-ain.com/article/egypt-economy-2019-world-leaders-investors (8) الحرة، الهيئة العامة للاستثمار والمناطق. 2019م. ترتيب مصر في التقارير الدولية. تاريخ الاسترجاع: أكتوبر 2019م. نشر .موقع: https://www.gafi.gov.eg/Arabic/whyegypt/Pages/Egypt-Ranking.aspx

Bank, T. W. (2019). Doing Business 2019. International Bank for Reconstruction and Development. (9) Bank, T. W. (2020). Doing Business 2020. International Bank for Reconstruction and Development. (10) (11) الحرة، الهيئة العامة للاستثمار والمناطق. 2019م. ترتيب مصر في التقارير الدولية. مرجع سابق ذكره.

Economics, T. (n.d.). Egypt - Credit Rating. Retrieved from https://tradingeconomics.com/egypt/rating (12) (13) الدولي، البنك. 2020م. كورونا كوي بالاقتصاد العالمي في غمرة أسوأ كساد منذ الحرب العالمية الثانية. تاريخ الاسترجاع: 8https://www.albankaldawli.org/ar/news/press-release/2020/06/08/covid-19-to- يونيو -2020م. plunge-global-economy-into-worst-recession-since-world-war-ii\#_ftn1 El-Khishin, S. (2020, April). Countermeasures for the COVID-19 Outbreak in Egypt: This Time is Really (14) Different. The Economic Research Forum, p: 1-5. 1-5. :OECD. (2020, April). Op.cit. P (15) Economics, T. (n.d.). Egypt GDP Annual Growth Rate. Retrieved from (16) https://tradingeconomics.com/egypt/gdp-growth-annual

Ibid. (17) 
(18) الدولي، البنك. 2019م. أرصدة ديون البلدان النامية ارتفعت إلى 7.8 تريليون دولار في 2018: إحصاءات البنك الدولي عن

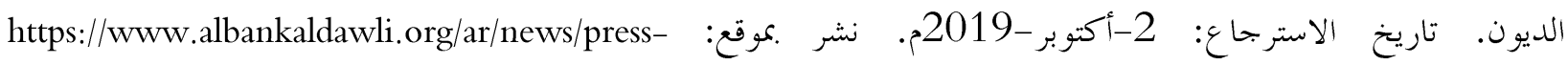
release/2019/10/02/debt-stocks-of-developing-countries-rose-to-78-trillion-in-2018-world-bankinternational-debt-statistics

(19) عبد الغفار، عبد الغفار فاروق. الدين العام الخارجي وسياسة تحويله لاستثمارات أجنبية دراسة الحالة المصرية. بجلة اقتصاديات شمال

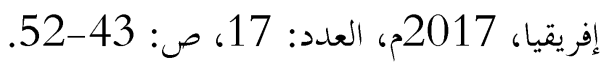

(20) الغايش، علياء محمد. أثار الديون الخارجية وإنعكاساتا على التنمية الاقتصادية في الدول النامية الحالة المصرية (خلال الفترة

2010- 2018). بحلة الشريعة و القانون. 2019م. العدد: 34، ص: 763-773.

Breisinger, C., Abdel Latif, A., Raouf, M., Wiebelt, M., (2020). COVID-19 and the Egyptian Economy (21) Estimating the impacts of expected reductions in tourism, Suez Canal revenues, and Remittances, International Food Policy Research Institute (IFPRI- Egypt), p: 1-6.

(22) حسني، خالد. 2020م. كيف تستخدم الحكومة المصرية قرض صندوق النقد الدولي؟: الاسواق العربية. تاريخ الاسترجاع: 2020

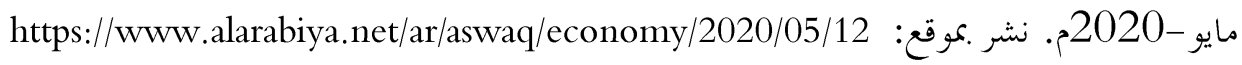
(23) ذكر الله، احمد. 2020م. تداعيات فيروس كورونا على الاقتصاد المصري: البوصلة. تاريخ الاسترجاع: 14-مارس-2020م. https://albosla.net نشر بموقع

IMF. (n.d.). ARAB REPUBLIC OF EGYPT. Retrieved from https://www.imf.org/en/Countries/EGY (24)

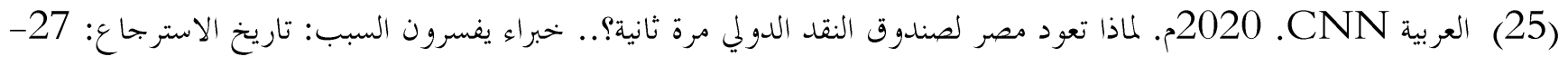

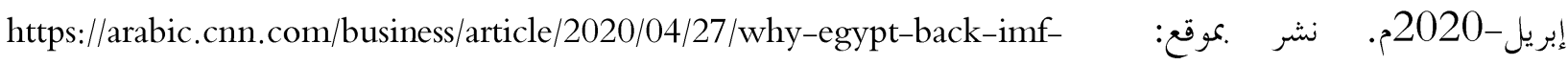
coronavirus 